\title{
Code Tracking Performance Analysis of GNSS Receivers with Blanking Model under Periodic Pulse Interference
}

\author{
Pengcheng $M A^{1}$, Xiaomei $T A N G^{1}$, Shengqiang $L O U^{1}$, Kuixing $L I U^{2}$, Gang $O U^{1}$ \\ ${ }^{1}$ College of Electronic Science and Technology, National University of Defense Technology, \\ Deya Road 109, Changsha, Hunan, 410073, China \\ ${ }^{2}$ Beijing Satellite Navigation Center, Yongfeng Road 5128, Haidian District, Beijing, 100000, China \\ mapengcheng1001@163.com, loushq@163.com
}

Submitted August 30, 2020 / Accepted February 3, 2021

\begin{abstract}
As one of the most common types of interference, pulse interference and its suppression methods have been widely studied, in which the blanking method consumes less hardware resources and it is more commonly used in the Global Navigation Satellite System (GNSS) receivers. Previous studies used carrier-to-noise ratio (CNR) as an indicator to evaluate the performance of the receiver after pulse suppression. However, CNR is not the most important parameter that should be measured. The main function of the GNSS receiver is to measure the distance between the satellite and the receiver. How the pulse interference with different periods and duty cycle affects the ranging performance of the navigation receiver after blanking has not been fully studied. This paper focuses on the influence of the blanking method on the code tracking performance. Through derivation and simulation, it can be found that the ranging accuracy of the delay locked loop may not be deteriorated sometimes when the blanking operation plays a role of narrow correlation and the frequency of the pulse interference is close to the pseudo code. However, the blanking algorithm will cause large code tracking deviation, which will seriously affect the ranging performance of the receiver.
\end{abstract}

\section{Keywords}

Pulse interference, pulse blanking, code tracking, delay locked loop, GNSS receiver

\section{Introduction}

GNSS can provide accurate positioning, navigation and timing service, and it plays an important role in many fields of military and daily life. However, due to the low power when the GNSS signal arrives at the receiver antenna, various types of interference, such as single-frequency interference [1], narrowband or broadband interference, multi-tone interference, pulse interference, have always been the threats for the receivers to providing trusted services. Among them, the pulse interference is mainly caused by the pulse signals falling into the L-band, such as the DME/TACAN system [2], the pseudo-satellite system [3] and the radar working near the L-band [4], which will affect the operation of the GNSS receivers seriously.

In order to suppress the influence of the pulse interference on GNSS receivers, there are many mitigating technologies being studied, such as nulling by array antennas, Analog-to-Digital Converter (ADC) limiting, interference reconstruction and cancellation, wavelet transform and time domain nulling algorithms, etc. The array antenna technique is a general method, which can be used not only to suppress the pulse interference, but also other broadband interferences [5]. However, the array antennas are not available for every GNSS receiver, and a large number of civil receivers are equipped with a single antenna. The method based on ADC amplitude limiting is easy to implement, but it may cause signal distortion when the pulse power is high [6]. The interference cancellation method reconstructs the interference signal locally by estimating the signal parameters, and then subtracts it from the received signal to achieve the purpose of eliminating interference. However, the interference pattern is usually unknown, and the propagation channel and other factors can easily lead to the decline of the performance of the cancellation method [7]. The pulse interference suppression method [8,9] based on wavelet transform increases the computation burden. The pulse blanking method has been widely used because of its simple implementation [10] and robust performance $[11,12]$, and just requires reasonable selection of relevant parameters and threshold [13-15].

For the pulse interference blanking algorithm, it is necessary to evaluate its impact on the navigation signal reception. Literature [16-19] evaluates the impact of pulse interference on the receiver based on the carrier-to-noise ratio. The effect of the blanking for pulse interference on navigation data demodulation is evaluated in [20]. The influence of the ideal time domain blanking on arrival of time (TOA) estimation under periodic pulse interference is analyzed in [21], based on [22, 23]. This literature comprehensively discusses nulling in time domain and frequency domain and also gives an equivalent noise power growth 
factor to calculate the equivalent TOA estimation error after blanking. The simulation results show that the signalto-noise ratio after blanking cannot be simply determined by duty cycle and that is also related to the pulse period and the time length of coherent accumulation. However, the influence on signal tracking deviation is not given in this reference when the pulse frequency is close to the pseudo code of GNSS signal. The influence analysis of pulse signal tracking accuracy is given in [24], but it is suitable for pseudo-satellite signal tracking scene, and pseudo-satellite signal generally transmits $[25,26]$ according to a certain pattern, which is not periodic in a period of time. Therefore, the model does not perfectly match the pulse interference blanking scenarios with different periods and duty cycles.

It can be seen from the current researches that the influence of pulse interference on the performance of the receiver mainly takes the $\mathrm{CNR}$ as the evaluation index. Moreover, the accuracy and deviation of the pseudo-range measurements are paid less attention, which are more important evaluation indicators for the receiver. In addition, a more accurate signal model is needed for the performance evaluation of the measurements generated by the receiver after pulse blanking. The main contribution of this paper is as follows: (1) a general signal model is established for assessing the influence of pulse interference on the performance of the receiver after blanking, and (2) the influence of different period and duty cycle on code tracking deviation is analyzed and different phenomenon is revealed which is given less consideration in the past work, and (3) the code tracking accuracy is studied under the pulse interference with different period and duty cycle when the GNSS receiver equipped with pulse blanking model, which extends the work in [24]. This work provides a theoretical reference for the performance evaluation of the pulse interference suppression methods.

The structure of this paper is as follows. The pulse blanking model and the input signal model are presented in Sec. 2. Section 3 introduces the theoretical analysis of the correlation value output. Then the blanking influence on code tracking accuracy and deviation is analyzed in Sec. 4. Simulation verification is discussed in Sec. 5. Finally, Section 6 summarizes the whole work.

\section{Signal Model}

\subsection{Pulse Blanking Model}

The ideal pulse blanking operation performs gate processing on the received navigation signal. This paper assumes that the pulse interference is periodic, and its period is $T_{\mathrm{s}}$. The duty cycle of the pulse is $d_{\mathrm{P}}$. The ideal pulse blanking operation has the same effect that the received signal is multiplied by the periodic square wave signal whose period is $T_{\mathrm{s}}$ and duty cycle is $d_{\mathrm{f}}=1-d_{\mathrm{P}}=\tau / T_{\mathrm{s}}$,

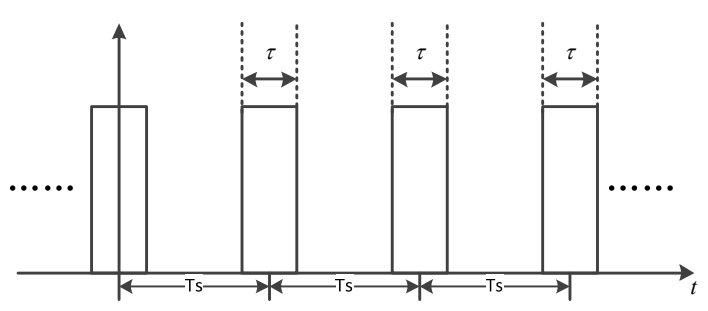

Fig. 1. Infinite periodic square wave.

where $\tau$ is the width of the square wave. The infinite periodic square wave signal is shown in Fig. 1.

The expression of the infinite periodic square wave signal is

$$
\begin{aligned}
g(t) & =g_{\tau}(t) * \delta_{T_{\mathrm{s}}}(t) \\
& =\sum_{n=-\infty}^{+\infty} g_{\tau}\left(t-n T_{\mathrm{s}}\right)
\end{aligned}
$$

where

$$
\begin{gathered}
g_{\tau}(t)=\left\{\begin{array}{cc}
1, & |t|<\frac{\tau}{2}, \\
0, & |t|>\frac{\tau}{2}
\end{array}\right. \\
\delta_{\mathrm{T}_{\mathrm{s}}}(t)=\sum_{n=-\infty}^{+\infty} \delta\left(t-n T_{\mathrm{s}}\right) .
\end{gathered}
$$

In this paper, the assumptive pulse period is not greater than the coherent integration time. In practical processing, the number of periodic pulse signals in a coherent integration period is limited, and its initial phase compared with the integral start time is random. The time domain expression of $N=T_{\mathrm{coh}} / T_{\mathrm{s}}$ pulse square signal is

$$
\begin{aligned}
g_{\mathrm{N}}(t) & =g_{\tau}(t)+g_{\tau}\left(t-T_{\mathrm{s}}\right)+\cdots+g_{\tau}\left(t-(N-1) T_{\mathrm{s}}\right) \\
& =\sum_{n=0}^{N-1} g_{\tau}\left(t-n T_{\mathrm{s}}\right) .
\end{aligned}
$$

There are mainly two cases (Phase I and Phase II) of the initial phase of the periodic pulse signal in an integration period $T_{\text {coh }}$ which is equal to one period of the pseudo code, for example, $1 \mathrm{~ms}$. Figure 2 shows a situation, when the square wave period $T_{\mathrm{s}}$ and the processing period $T_{\mathrm{coh}}$ are equal $\left(T_{\mathrm{s}}=T_{\mathrm{coh}}\right)$.

Among them, $T_{0}$ represents the initial phase of the pulse signal in the coherent integral period. The time domain expressions are respectively written as

$$
\begin{gathered}
g_{\tau 1}(t)=\left\{\begin{array}{ll}
1, & T_{0} \leq t<T_{0}+\tau \\
0, & \text { else }
\end{array}, 0 \leq T_{0}<T_{s}-\tau,\right. \\
g_{\tau 2}(t)=\left\{\begin{array}{ll}
1, & 0 \leq t<\tau-T_{\mathrm{s}}+T_{0} \\
0, & \text { or } T_{0} \leq t<T \\
0, & \tau-T_{\mathrm{s}}+T_{0} \leq t<T
\end{array}, \quad T_{\mathrm{s}}-\tau \leq T_{0}<T_{\mathrm{s}}\right.
\end{gathered}
$$

where $g_{\tau 1}(t)$ and $g_{\tau 2}(t)$ represent time domain wave of the pulse signal with two kinds of phases respectively. 


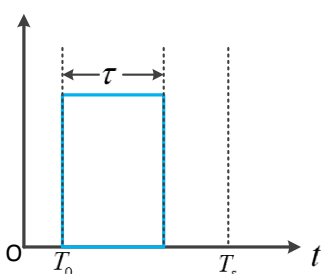

(a) Phase I

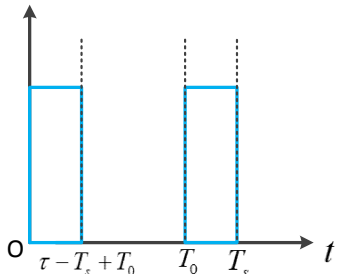

(b) Phase II
Fig. 2. Periodic pulse signals in a coherent integration period.

\subsection{Received Signal Model}

The incoming signal $x(t)$ can be expressed as

$$
x(t)=s(t)+n(t)
$$

where $s(t)$ represents the navigation signal, and it can be expressed as

$$
s(t)=\sqrt{2 P} c(t-\tau) D(t-\tau) \cos \left(2 \pi\left(f_{\mathrm{IF}}+f_{\mathrm{d}}\right) t+\theta\right)
$$

where $P$ represents the power of the navigation signal arriving at the receiving antenna. $c(t)$ is the pseudo code sequence and the code chip width is $T_{\mathrm{c}}$. The signal delay between the satellite and the receiver is $\tau$. $D$ represents the navigation message and $f_{\mathrm{IF}}$ is the carrier intermediate frequency. The Doppler frequency caused by the mutual movement between the satellite and the receiver is $f_{\mathrm{d}}$, and $\theta$ is the initial carrier phase. $n(t)$ is a narrow-band Gaussian white noise random process, and the power spectral density is $N_{0} / 2$ and the autocorrelation function is approximately as $R_{\mathrm{n}}(\tau)=\delta(\tau) N_{0} / 2$.

In practice, multiple satellite signals will be received. This paper takes one satellite as an example for analysis. After the ideal pulse blanking operation, the input signal model can be expressed as

$$
x_{\mathrm{g}}(t)=g_{\mathrm{N}}(t) x(t)=g_{\mathrm{N}}(t) s(t)+g_{\mathrm{N}}(t) n(t) .
$$

\section{Output of the Integrator}

In the process of acquisition and tracking, it is necessary to locally generate pseudo code and reference carrier and multiply with the received signal, and then integrate multiplication results to obtain the correlation measurements. In this paper, the influence of the band-pass filter in radio frequency (RF) front-end is ignored, and the output result of the integral processing is analyzed after the received signal being blanked by ideal pulse. The structure of the integral processing flow is shown in Fig. 3.

Among them, the reference signals of the in-phase and the orthogonal branch are respectively shown as follows

$$
\begin{aligned}
& l c(t)=\sqrt{2} c(t-\hat{\tau}) \cos \left(2 \pi\left(f_{\mathrm{IF}}+\hat{f}_{\mathrm{d}}\right) t\right), \\
& l s(t)=\sqrt{2} c(t-\hat{\tau}) \sin \left(2 \pi\left(f_{\mathrm{IF}}+\hat{f}_{\mathrm{d}}\right) t\right) .
\end{aligned}
$$

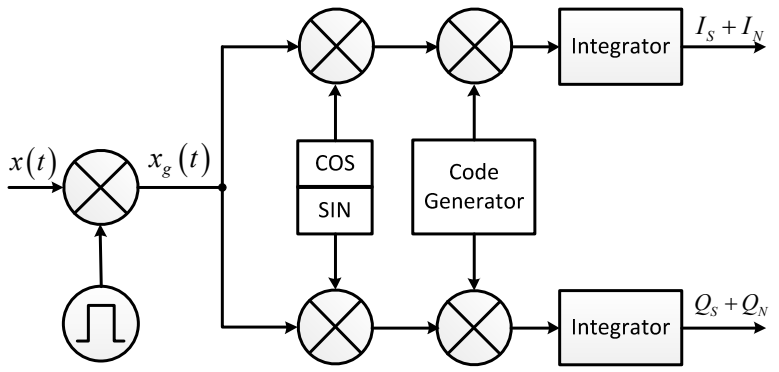

Fig. 3. The structure of the integral processing flow.

\subsection{Signal Component}

When the periodic pulse acts on the received navigation signal, according to the time length of the pulse period, it can be divided into two cases: the pulse period is close to the integration time $T_{\text {coh }}$ and close to the pseudo code chip $T_{\mathrm{c}}$. The pulse period of the latter is much smaller than that of the first case.

It is assumed that the message symbol does not change during the integration. When the period is close to the coherent integral time, the output results of the in-phase branch of the integrator is shown as (12)

$$
\begin{aligned}
I_{\mathrm{S}} & =\frac{1}{T_{\text {coh }}} \int_{0}^{T_{\text {cob }}}\left[\begin{array}{c}
g_{\mathrm{N}}(t) \sqrt{2 P} c(t-\tau) \times \\
\cos \left(2 \pi\left(f_{\mathrm{IF}}+f_{\mathrm{d}}\right) t+\theta\right) \times l c(t)
\end{array}\right] \mathrm{d} t \\
& =\frac{\sqrt{P}}{T_{\text {coh }}} \int_{0}^{d_{\mathrm{f}} T_{\mathrm{coh}}} c(t-\tau) c(t-\hat{\tau}) g_{\mathrm{N}}(t) \cos \left(2 \pi f_{\mathrm{e}} t+\theta\right) \mathrm{d} t \\
& =\frac{d_{\mathrm{f}} \sqrt{P} R\left(\tau_{\mathrm{e}}\right)}{T_{\text {coh }}} \sin c\left(\pi f_{\mathrm{e}} T_{\text {coh }}\right) \cos \left(2 \pi f_{\mathrm{e}}\left(\frac{T_{\text {coh }}}{2}\right)+\theta\right) .
\end{aligned}
$$

Among them, $f_{\mathrm{e}}=f_{\mathrm{d}}-\hat{f}_{\mathrm{d}}, \tau_{\mathrm{e}}=\tau-\hat{\tau} . R\left(\tau_{\mathrm{e}}\right)$ is the autocorrelation function of the normalized spread spectrum code, and its expression is

$$
R\left(\tau_{\mathrm{e}}\right)= \begin{cases}1-\frac{\left|\tau_{\mathrm{e}}\right|}{T_{\mathrm{c}}} & \left|\tau_{\mathrm{e}}\right|<T_{\mathrm{c}} \\ 0 & \text { else }\end{cases}
$$

According to the same deduction, the coherent integral value of the orthogonal branch can be deduced as (14).

$$
Q_{\mathrm{S}}=\frac{d_{\mathrm{f}} \sqrt{P} R\left(\tau_{\mathrm{e}}\right)}{T_{\text {coh }}} \sin c\left(\pi f_{\mathrm{e}} T_{\mathrm{coh}}\right) \sin \left(2 \pi f_{\mathrm{e}}\left(\frac{T_{\mathrm{coh}}}{2}\right)+\theta\right) \text {. }
$$

When the pulse period is close to the code chip $T_{\mathrm{c}}$, the correlation between the pulse and the carrier can be ignored, and the integral output of the in-phase branch can be deduced as (15).

$$
\begin{aligned}
I_{\mathrm{S}} & =\frac{1}{T_{\text {coh }}} \int_{0}^{T_{\text {coh }}} g_{\mathrm{N}}(t) \sqrt{2 P} c(t-\tau) \cos \left(2 \pi\left(f_{\mathrm{IF}}+f_{\mathrm{d}}\right) t+\theta\right) l c(t) \mathrm{d} t \\
& =\frac{\sqrt{P}}{T_{\text {coh }}} \int_{0}^{T_{\text {coh }}} g_{\mathrm{N}}(t) c(t-\tau) c(t-\hat{\tau}) \cos \left(2 \pi f_{\mathrm{e}} t+\theta\right) \mathrm{d} t \\
& \approx \frac{\sqrt{P}}{T_{\text {coh }}} \int_{0}^{T_{\text {coh }}} g_{\mathrm{N}}(t) c(t-\tau) c(t-\hat{\tau}) \mathrm{d} t \int_{0}^{T_{\text {coh }}} \cos \left(2 \pi f_{\mathrm{e}} t+\theta\right) \mathrm{d} t .
\end{aligned}
$$


As mentioned earlier, the periodic pulse square wave has two phase forms $g_{\tau 1}(t)$ and $g_{\tau 2}(t)$, and the normalization correlation functions after multiplying and accumulating with the local pseudo code are respectively expressed as follows.

$$
\begin{aligned}
& R_{\mathrm{g} 1}\left(\tau_{\mathrm{e}}\right)=\int_{-\infty}^{\infty} \sum_{n=0}^{N-1} g_{\tau 1}\left(t-n T_{\mathrm{s}}\right) c(t-\tau) c(t-\hat{\tau}) \mathrm{d} t, \\
& R_{\mathrm{g} 2}\left(\tau_{\mathrm{e}}\right)=\int_{-\infty}^{\infty} \sum_{n=0}^{N-1} g_{\tau 2}\left(t-n T_{\mathrm{s}}\right) c(t-\tau) c(t-\hat{\tau}) \mathrm{d} t .
\end{aligned}
$$

Taking the pulse square wave period $T_{\mathrm{s}}=T_{\mathrm{c}}$ and duty cycle of $50 \%$ as an example, the time domain waveforms of the two forms are illustrated in Fig. 4.

The periodic pulse waveforms of the two phases are different in the processing with the original pseudo codes. The pulse waveform of the first kind phase eliminates the edges of the code, while the second kind retains them. The edge of the chip has the characteristic of high frequency because it changes more sharply than the rest of the code. The high-frequency component represents the better tracking accuracy [22]. When the coherent integration time is not an integral multiple of the pulse period, parts of the edges are retained and parts of them are suppressed. Ranging performance under this situation is between complete suppression and retention. The pulse waveform of the first kind phase represents the situation of suppression and the other represents retention. Only two extreme cases are analyzed here. After derivation, the correlation results under the two forms are respectively shown as (18) and (19).

$$
\begin{aligned}
& R_{\mathrm{g} 1}\left(\tau_{\mathrm{e}}\right)= \begin{cases}0, & \tau_{\mathrm{e}} \leq-T_{\mathrm{s}}+T_{0} \\
\tau_{\mathrm{e}}+T_{\mathrm{s}}-T_{0} & -T_{\mathrm{s}}+T_{0}<\tau_{\mathrm{e}} \leq-T_{\mathrm{s}}+T_{0}+d_{\mathrm{f}} T_{\mathrm{s}} \\
d_{\mathrm{f}} T_{\mathrm{s}} & -T_{\mathrm{s}}+T_{0}+d_{\mathrm{f}} T_{\mathrm{s}}<\tau_{\mathrm{e}} \leq T_{0} \\
-\tau_{\mathrm{e}}+d_{\mathrm{f}} T_{\mathrm{s}}+T_{0} & T_{0}<\tau_{\mathrm{e}} \leq T_{0}+d_{\mathrm{f}} T_{\mathrm{s}} \\
0 & T_{0}+d_{\mathrm{f}} T_{\mathrm{s}}<\tau_{\mathrm{e}}\end{cases} \\
& R_{\mathrm{g} 2}\left(\tau_{\mathrm{e}}\right)= \begin{cases}0, & \tau_{\mathrm{e}} \leq-T_{\mathrm{s}} \\
\tau_{\mathrm{e}}+T_{\mathrm{s}}, & -T_{\mathrm{s}}<\tau_{\mathrm{e}} \leq-2 T_{\mathrm{s}}+T_{0}+d_{\mathrm{f}} T_{\mathrm{s}} \\
d_{\mathrm{f}} T_{\mathrm{s}}-T_{\mathrm{s}}+T_{0}, & -2 T_{\mathrm{s}}+T_{0}+d_{\mathrm{f}} T_{\mathrm{s}}<\tau_{\mathrm{e}} \leq-T_{\mathrm{s}}+T_{0} \\
\tau_{\mathrm{e}}+d_{\mathrm{f}} T_{\mathrm{s}}, & -T_{\mathrm{s}}+T_{0}<\tau_{\mathrm{e}} \leq 0 \\
-\tau_{\mathrm{e}}+d_{\mathrm{f}} T_{\mathrm{s}}, & 0<\tau_{\mathrm{e}} \leq-T_{\mathrm{s}}+T_{0}+d_{\mathrm{f}} T_{\mathrm{s}} \\
T_{\mathrm{s}}-T_{0}, & -T_{\mathrm{s}}+T_{0}+d_{\mathrm{f}} T_{\mathrm{s}}<\tau_{\mathrm{e}} \leq T_{0} \\
-\tau_{\mathrm{e}}+T_{\mathrm{s}}, & T_{0}<\tau_{\mathrm{e}} \leq T_{\mathrm{s}} \\
0 & T_{\mathrm{s}}<\tau_{\mathrm{e}}\end{cases}
\end{aligned}
$$

The correlation function is shown in Fig. 5. In Fig. 5, Phase I and Phase II represent the autocorrelation results of two phases respectively, and label of $T_{\mathrm{s}}=T_{\text {coh }}$ represents the autocorrelation results with larger pulse period $T_{\text {coh }}$. These four correlation peaks are obviously different. It can be seen that the change of the correlation peak is half of the original BPSK signal which is due to the result of the pulse signal with a duty cycle of $50 \%$. Based on a simple derivation, the in-phase correlation values of Phase I and II can be respectively expressed as

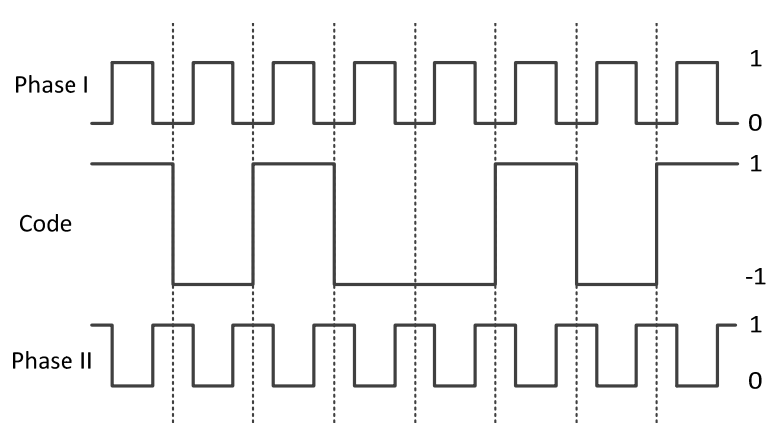

Fig. 4. Time domain waveforms of the two kinds of phases.

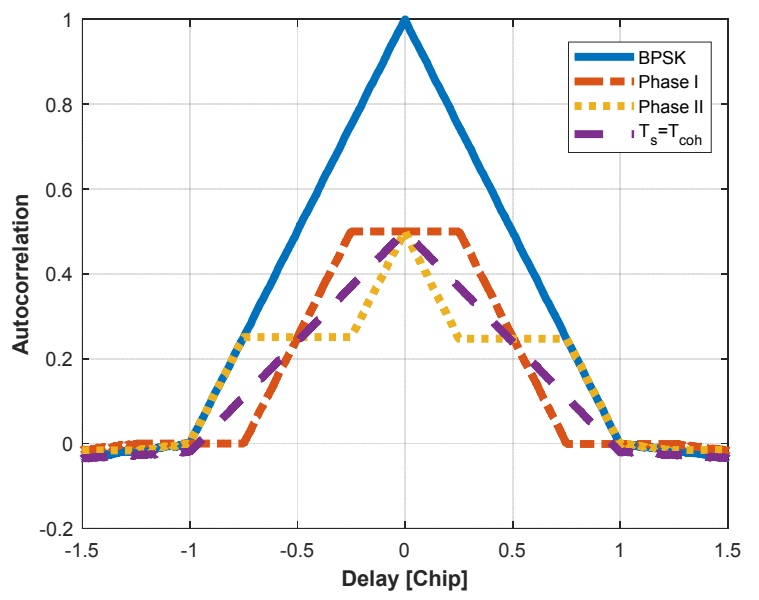

Fig. 5. Correlation function.

$I_{\mathrm{S} 1}=\frac{1}{T_{\text {coh }}} \sqrt{P} R_{\mathrm{g} 1}\left(\tau_{\mathrm{e}}\right) \sin c\left(\pi f_{\mathrm{e}} T_{\mathrm{coh}}\right) \cos \left(2 \pi f_{\mathrm{e}}\left(\frac{T_{\mathrm{coh}}}{2}\right)+\theta\right)$,

$I_{\mathrm{S} 2}=\frac{1}{T_{\text {coh }}} \sqrt{P} R_{\mathrm{g} 2}\left(\tau_{\mathrm{e}}\right) \sin c\left(\pi f_{\mathrm{e}} T_{\mathrm{coh}}\right) \cos \left(2 \pi f_{\mathrm{e}}\left(\frac{T_{\mathrm{coh}}}{2}\right)+\theta\right)$.

In the same way, the correlation values of orthogonal branches can be respectively deduced as follows:

$$
\begin{aligned}
& Q_{\mathrm{S} 1}=\frac{1}{T_{\mathrm{coh}}} \sqrt{P} R_{\mathrm{g} 1}\left(\tau_{\mathrm{e}}\right) \sin c\left(\pi f_{\mathrm{e}} T_{\mathrm{coh}}\right) \sin \left(2 \pi f_{\mathrm{e}}\left(\frac{T_{\mathrm{coh}}}{2}\right)+\theta\right), \\
& Q_{\mathrm{S} 2}=\frac{1}{T_{\text {coh }}} \sqrt{P} R_{\mathrm{g} 2}\left(\tau_{\mathrm{e}}\right) \sin c\left(\pi f_{\mathrm{e}} T_{\mathrm{coh}}\right) \sin \left(2 \pi f_{\mathrm{e}}\left(\frac{T_{\mathrm{coh}}}{2}\right)+\theta\right) .
\end{aligned}
$$

\subsection{Noise Component}

The expression of in-phase branch when the Gaussian narrowband noise passes through the integrator is shown as (24)

$$
I_{\mathrm{N}}=\frac{1}{T_{\text {coh }}} \int_{0}^{T_{\mathrm{coh}}} g_{\mathrm{N}}(t) n(t) c\left(t-\hat{\tau}_{\mathrm{A}}\right) \mathrm{d} t
$$

As $n(t)$ is a zero-mean Gaussian narrowband random process, $I_{\mathrm{N}}$ is also a zero-mean Gaussian narrowband random process after linear processing. It can be assumed that narrowband noise, periodic pulse signals and pseudo code sequences are pairwise uncorrelated. According to the conclusion of [22], the variance of the correlation value is derived as 


$$
\operatorname{Var}\left(I_{\mathrm{N}}\right)=\operatorname{Var}\left(I_{\mathrm{Q}}\right) \approx \frac{1}{T_{\text {coh }}} \int_{-\infty}^{\infty} R_{\mathrm{g}}(\tau) R_{\mathrm{n}}(\tau) R(\tau) \mathrm{d} \tau=\frac{d_{\mathrm{f}} N_{0}}{2 T_{\text {coh }}}
$$

where $R_{\mathrm{g}}(\tau)$ represents the autocorrelation function of $g_{\mathrm{N}}(t)$ and its expression is shown as

$$
R_{\mathrm{g}}(\tau)= \begin{cases}1-\frac{|\tau|}{d_{\mathrm{f}} T_{\mathrm{s}}} & |\tau|<d_{\mathrm{f}} T_{\mathrm{s}} \\ 0 & \text { else }\end{cases}
$$

\section{Code Tracking Performance}

Code tracking performance can be evaluated by tracking accuracy and deviation. The tracking accuracy is mainly evaluated by the variance under unbiased tracking, and the tracking deviation is mainly reflected by the zerocrossing offset of the S-curve. The slope of S-curve also affects the tracking accuracy. The tracking loop adopted in this paper is a general non-coherent early-late pseudo code tracking loop. The interval between early and late correlator is $d$ chip. The tracking loop architecture is shown as Fig. 6.

For the sake of convenience, the output of each integrator can be expressed as

$$
\begin{gathered}
y_{\mathrm{IE}}=I_{\mathrm{SE}}+I_{\mathrm{NE}}, \\
y_{\mathrm{QE}}=Q_{\mathrm{SE}}+Q_{\mathrm{NE}}, \\
y_{\mathrm{IL}}=I_{\mathrm{SL}}+I_{\mathrm{NL}}, \\
y_{\mathrm{QL}}=Q_{\mathrm{SL}}+Q_{\mathrm{NL}} .
\end{gathered}
$$

Code tracking performance analysis is divided into two parts: coherent processing and non-coherent processing.

\subsection{Coherent Processing}

It is assumed that there is no tracking error in the carrier phase and frequency, and the coherent processing represents the upper limit of the performance of the code tracking loop. The discriminator expression is

$$
e(k)=y_{\mathrm{IL}}-y_{\mathrm{IE}} \text {. }
$$

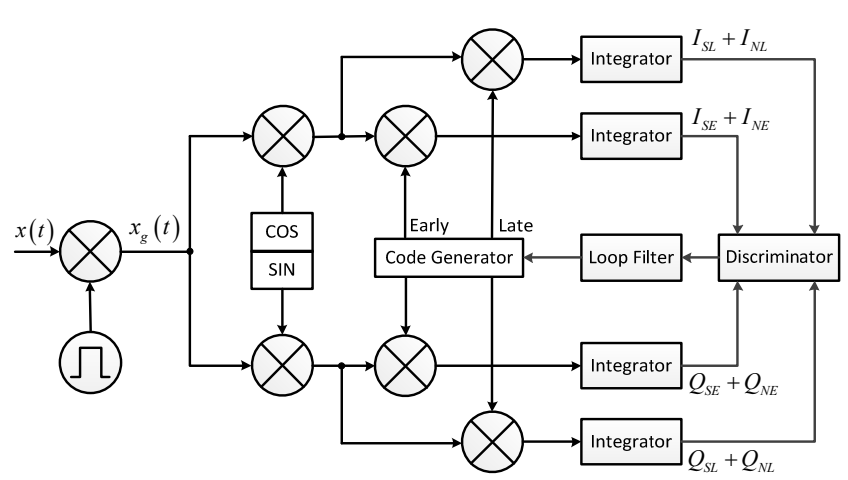

Fig. 6. The code tracking loop architecture.
When the code phase error is small and near zero, the discriminator can be linearized and its expression is

$$
\begin{aligned}
e(k) & =\sqrt{P}\left[R\left(\tau_{\mathrm{e}}-\frac{d}{2}\right)-R\left(\tau_{\mathrm{e}}+\frac{d}{2}\right)\right]+I_{\mathrm{NE}}-I_{\mathrm{NL}} \\
& \approx \sqrt{P} K \tau_{\mathrm{e}}+I_{\mathrm{NE}}-I_{\mathrm{NL}}
\end{aligned}
$$

where the noise component can be deduced as

$$
\begin{aligned}
I_{\mathrm{NE}}-I_{\mathrm{NL}} & =\frac{1}{T_{\text {coh }}} \int_{0}^{T_{\text {coh }}} g_{\mathrm{N}}(t) n(t) c\left(t-\frac{d}{2}\right) \mathrm{d} t \\
& -\frac{1}{T_{\text {coh }}} \int_{0}^{T_{\text {coh }}} g_{\mathrm{N}}(t) n(t) c\left(t+\frac{d}{2}\right) \mathrm{d} t \\
& =\frac{1}{T_{\text {coh }}} \int_{0}^{T_{\text {coh }}} g_{\mathrm{N}}(t) n(t)\left(c\left(t-\frac{d}{2}\right)-c\left(t+\frac{d}{2}\right)\right) \mathrm{d} t \\
& =\frac{1}{T_{\text {coh }}} \int_{0}^{T_{\text {coh }}} g_{\mathrm{N}}(t) n(t) c_{\mathrm{d}}(t) \mathrm{d} t .
\end{aligned}
$$

When the pulsed period approaches the integration time, the above equation can be transformed into

$$
w=I_{\mathrm{NE}}-I_{\mathrm{NL}}=\frac{1}{T_{\text {coh }}} \int_{0}^{d_{\mathrm{r}} T_{N}^{N}} n(t) c_{\mathrm{d}}(t) \mathrm{d} t .
$$

The power spectral density of the noise in the loop can be represented by the $S_{\mathrm{w}}(f)$ when $f=0$ as the loop bandwidth is narrow compared with the noise, which can be deduced as

$$
S_{\mathrm{w}}(0)=d_{\mathrm{f}} N_{0}[1-R(d)] .
$$

When the pulse period is close to the pseudo code chip, the noise term $w=I_{\mathrm{NE}}-I_{\mathrm{NL}}$ is different, and its variance is affected by the initial phase of the pulse and the correlation interval, which can be illustrated in Fig. 7.

In Fig. 7, $c_{\mathrm{d}}(t)=c(t-d / 2)-c(t+d / 2)$, and $g_{\mathrm{N} 1}(t)$, $g_{\mathrm{N} 2}(t), g_{\mathrm{N} 3}(t)$ represent three types of pulse signal with different duty cycles. The power of the noise depends on the non-zero range of the product of $g_{\mathrm{N}}(t)$ and $c_{\mathrm{d}}(t)$. In other words, the power of the noise term is limited by the pulse duty cycle when the non-zero part of a pulse period is less than the correlation interval, while when the non-zero

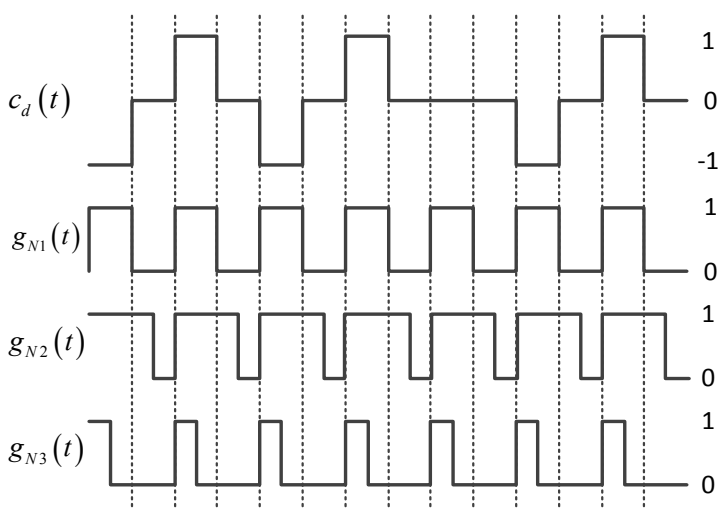

Fig. 7. The noise is leaked through the non-zero part of $g_{\mathrm{N}}(t) c_{\mathrm{d}}(t)$. 
part of a period is larger than the correlation interval, the power of the noise term is limited by the correlation interval. Therefore, when the correlator interval is greater than or equal to the duty cycle part, the power spectral density of the noise can be deduced as

$$
S_{\mathrm{w}}(0)=d_{\mathrm{f}} N_{0}[1-R(d)] .
$$

When the correlator interval is small and is less than the duty cycle, the power spectral density of the noise can be expressed as

$$
S_{\mathrm{w}}(0)=N_{0}[1-R(d)] \text {. }
$$

For the phase discrimination gain $K$, there are many literatures to calculate it theoretically $[22,24]$. The variance of tracking error by coherent processing can be expressed as (38)

$$
\sigma_{\tau_{\mathrm{e}-\mathrm{Coh}}}^{2} \approx \frac{2 B_{\mathrm{L}} S_{\mathrm{w}}(0)}{K^{2} P}
$$

\subsection{Non-Coherent Processing}

The assumption that there is no tracking error in the carrier phase in the above section is unrealistic and a noncoherent early-late discriminator is always used to overcome this problem. The non-coherent early-late discriminator can be defined as

$$
e(k)=\left[y_{\mathrm{IL}}^{2}+y_{\mathrm{QL}}^{2}\right]-\left[y_{\mathrm{IE}}^{2}+y_{\mathrm{QE}}^{2}\right] \text {. }
$$

It is assumed that the frequency of the carrier has been tracked accurately at this time, which means $f_{\mathrm{e}}=0$. Assume that the tracking error of the code phase is very small and the above formula can be linearized to

$$
\begin{aligned}
e(k) & =P\left[R^{2}\left(\tau_{\mathrm{e}}-\frac{d}{2}\right)-R^{2}\left(\tau_{\mathrm{e}}+\frac{d}{2}\right)\right]+n_{1}+n_{2} \\
& \approx P K \tau_{\mathrm{e}}+n_{1}+n_{2}
\end{aligned}
$$

where $K, n_{1}, n_{2}$ represent

$$
\begin{gathered}
K=\left.\frac{\mathrm{d}}{\mathrm{d} \tau_{\mathrm{e}}}\left[R^{2}\left(\tau_{\mathrm{e}}-\frac{d}{2}\right)-R^{2}\left(\tau_{\mathrm{e}}+\frac{d}{2}\right)\right]\right|_{\tau_{\mathrm{e}}=0}, \\
n_{1}=\left(I_{\mathrm{NL}}^{2}+Q_{\mathrm{NL}}^{2}\right)-\left(I_{\mathrm{NE}}^{2}+Q_{\mathrm{NE}}^{2}\right), \\
n_{2}=2 \sqrt{P}\left[R\left(\tau_{\mathrm{e}}-\frac{d}{2}\right) I_{\mathrm{NE}}-R\left(\tau_{\mathrm{e}}+\frac{d}{2}\right) I_{\mathrm{NL}}\right] .
\end{gathered}
$$

When the pulse period approaches the integration time $T_{\text {coh }}$, according to the derivation method in [24], the variance of the above two noise components $n_{1}$ and $n_{2}$ can be respectively deduced as

$$
\begin{gathered}
\operatorname{var}\left(n_{1}\right)=2 d_{\mathrm{f}}^{2} \frac{N_{0}^{2}}{T_{\text {coh }}^{2}}\left[1-R^{2}(d)\right], \\
\operatorname{var}\left(n_{2}\right)=4 d_{\mathrm{f}}^{3} P R^{2}\left(\frac{d}{2}\right) \frac{N_{0}}{T_{\text {coh }}}[1-R(d)] .
\end{gathered}
$$

However, when the pulse period is close to the pseudo code chip $T_{\mathrm{c}}$, the noise components $n_{2}$ is related to correlator spacing. When the correlator interval is larger than the pulse duty cycle time, the variance of $n_{2}$ can be deduced as

$$
\operatorname{var}\left(n_{2}\right)=4 P d_{\mathrm{f}} R_{\mathrm{g} 1}^{2}\left(\frac{d}{2}\right) \frac{N_{0}}{T_{\text {coh }}}[1-R(d)] .
$$

When the correlator interval is less than the pulse duty cycle time, the variance of $n_{2}$ can be deduced as

$$
\operatorname{var}\left(n_{2}\right)=4 P R_{\mathrm{g} 2}^{2}\left(\frac{d}{2}\right) \frac{N_{0}}{T_{\text {coh }}}[1-R(d)] .
$$

Assuming that the code tracking bandwidth is very small compared to the narrowband noise, the power spectral density of the noise in the code tracking loop at $f_{\mathrm{e}}=0$ can be expressed as

$$
S_{\mathrm{N}}(0) \approx\left[\operatorname{var}\left(n_{1}\right)+\operatorname{var}\left(n_{2}\right)\right] T_{\text {coh }} .
$$

To sum up, when the pulse period approaches the integration time, the variance of code tracking loop error caused by thermal noise can be deduced as

$$
\begin{aligned}
\sigma_{\tau_{\mathrm{c}-\mathrm{NCOh}}}^{2} & \approx \frac{2 B_{\mathrm{L}} S_{\mathrm{N}}(0)}{K^{2} P^{2}} \\
& =\frac{2 d_{\mathrm{f}}^{3} B_{\mathrm{L}} N_{0}}{K^{2} P}\left[4 R^{2}\left(\frac{d}{2}\right)(1-R(d))+\frac{2 d_{\mathrm{f}} N_{0}}{P T_{\text {coh }}}\left(1-R^{2}(d)\right)\right] .
\end{aligned}
$$

When the pulsed period is close to the pseudo code period and the correlator interval is larger than the pulse duty cycle time, the expression of the code tracking loop error deduced as

$$
\begin{aligned}
\sigma_{\tau_{\mathrm{c}-\mathrm{NCOh}}}^{2} & \approx \frac{2 B_{\mathrm{L}} S_{\mathrm{N}}(0)}{K^{2} P^{2}} \\
& =\frac{2 d_{\mathrm{f}} B_{\mathrm{L}} N_{0}}{K^{2} P}\left[4 R_{\mathrm{g} 1}^{2}\left(\frac{d}{2}\right)(1-R(d))+\frac{2 d_{\mathrm{f}} N_{0}}{P T_{\text {coh }}}\left(1-R^{2}(d)\right)\right] .
\end{aligned}
$$

What's more, when the correlator interval is less than the pulse duty cycle time, the variance of code tracking loop error caused by thermal noise can be deduced as

$$
\begin{aligned}
\sigma_{\tau_{\mathrm{c} \_} \mathrm{NCoh}}^{2} & \approx \frac{2 B_{\mathrm{L}} S_{\mathrm{N}}(0)}{K^{2} P^{2}} \\
& =\frac{2 B_{\mathrm{L}} N_{0}}{K^{2} P}\left[4 R_{\mathrm{g} 2}^{2}\left(\frac{d}{2}\right)(1-R(d))+\frac{2 d_{\mathrm{f}}^{2} N_{0}}{P T_{\text {coh }}}\left(1-R^{2}(d)\right)\right] .
\end{aligned}
$$

In addition, another important tool for evaluating the performance of code tracking is the S-curve, which can be defined as

$$
S\left(\tau_{\mathrm{e}}\right)=R^{2}\left(\tau_{\mathrm{e}}-\frac{d}{2}\right)-R^{2}\left(\tau_{\mathrm{e}}+\frac{d}{2}\right) .
$$

For example, the slopes $K$ of three typical scenarios can be expressed in Tab. 1 . 


\begin{tabular}{|c|c|c|c|c|}
\hline No & Pulse period & Pulse phase & $\boldsymbol{d}$ & Slope of S-curve \\
\hline 1 & $T_{\mathrm{coh}} / 2$ & Phase I / Phase II & 1 & $4 d_{\mathrm{f}}^{2}(1-d / 2)$ \\
\hline 2 & $T_{\mathrm{c}}$ & Phase I & 1 & $-2 d+2 T_{\mathrm{s}}\left(1+d_{\mathrm{f}}\right)$ \\
\hline 3 & $T_{\mathrm{c}}$ & Phase II & $1 / 8$ & $4\left(d_{\mathrm{f}} T_{\mathrm{s}}-d / 2\right)$ \\
\hline
\end{tabular}

Tab. 1. Slopes of three typical scenarios.

The tracking deviation of the pseudo code phase can be directly obtained according to the offset between the zero-crossing point and the origin of the S-curve, and the ranging deviation $\tau_{\mathrm{e}}$ can be expressed as

$$
\tau_{\mathrm{e}} \mid S\left(\tau_{\mathrm{e}}\right)=0
$$

When the pulse period is close to the pseudo code chip, it can be seen from the previous analysis that the shape of the correlation functions of the two kinds of phase has changed obviously. They are no longer a normal triangle. This leads to a great change in the ranging performance of the receiver.

\section{Simulation}

In order to analyze and verify the effects of the pulse period, duty cycle and pulse phase on code tracking performance, S-curve characteristics and unbiased tracking accuracy of non-coherent processing are carried out by simulation. The information about the used hardware and software equipment utilized for simulation is shown in Tab. 2.

\subsection{S-curve}

When the receiver is handed over from the acquisition stage to the tracking stage, a larger correlator interval is usually adopted at the beginning to obtain a larger traction range, and then the correlator interval is gradually reduced to obtain higher tracking accuracy. Therefore, this paper selects the commonly used $1 / 2$ and $1 / 16$ chip as correlator intervals to analyze the influence on the S-curve under the conditions of different periods, duty cycle and initial phase of the pulse. The simulation flow chart is shown as Fig. 8.

When the correlator interval is $1 / 2$ chip, the pulse period is $T_{\mathrm{s} 0}=T_{\text {coh }}=1 \mathrm{~ms}, T_{\mathrm{s} 1}=T_{\mathrm{c}}$ and $T_{\mathrm{s} 2}=T_{\mathrm{c}} / 2$, and the duty cycle is $25 \%, 50 \%$ and $75 \%$ respectively, the S-curves of two types of pulse phase are shown from Fig. 9 to Fig. 14.

As can be seen from Fig. 9 to Fig. 14, the pulse phase types, pulse frequency and duty cycle have a great influence on the S-curve. First of all, the duty cycle directly leads to the decrease of the slope of the S-curve, and the smaller the duty cycle is, the smaller the slope is. Secondly, there is little ranging deviation when the pulse period is close to the coherent integration time no matter what the pulse phase is, while it will produce a larger ranging deviation when the pulse period is consistent with the code chip width and the pulse can suppress most of the high frequency part of the pseudo code. What's more, when there is

\begin{tabular}{|c|c|c|}
\hline No & Equipment & Information \\
\hline \multirow{2}{*}{1} & & MagicBook 2019 \\
& PC configuration & CPU: AMD Ryzen 7 3700U \\
& & RAM: 8GB \\
\hline 2 & Software & MATLAB 2018a \\
\hline
\end{tabular}

Tab. 2. Information about the used hardware and software equipment utilized for simulation.

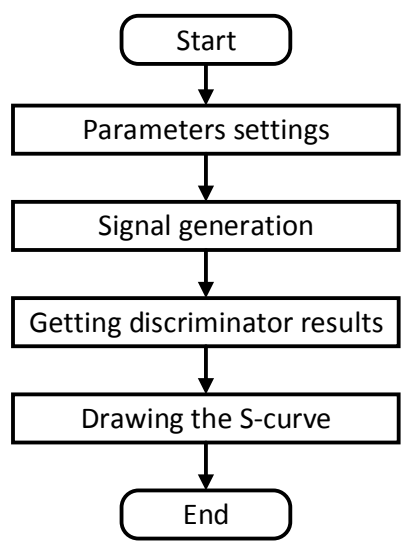

Fig. 8. The simulation flow chart of S-curve.

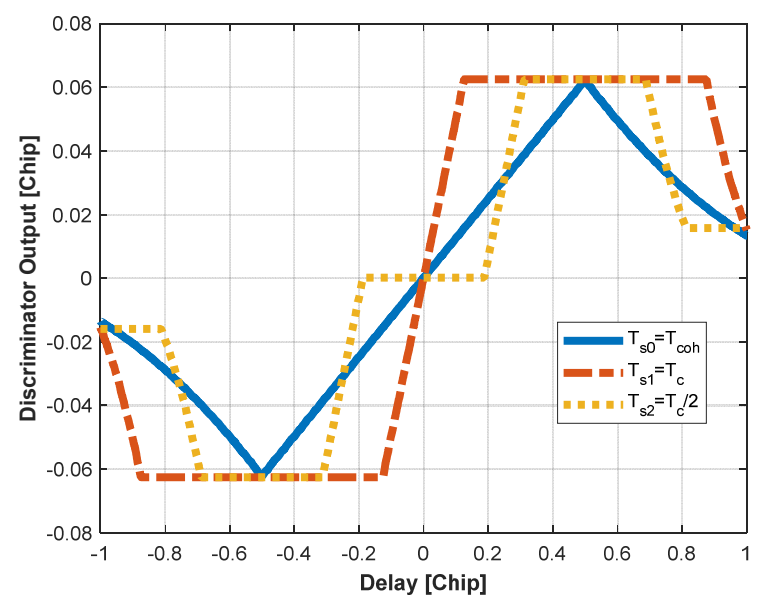

Fig. 9. S-curve of the code tracking loop when $d=1, d_{\mathrm{f}}=25 \%$, Phase I.

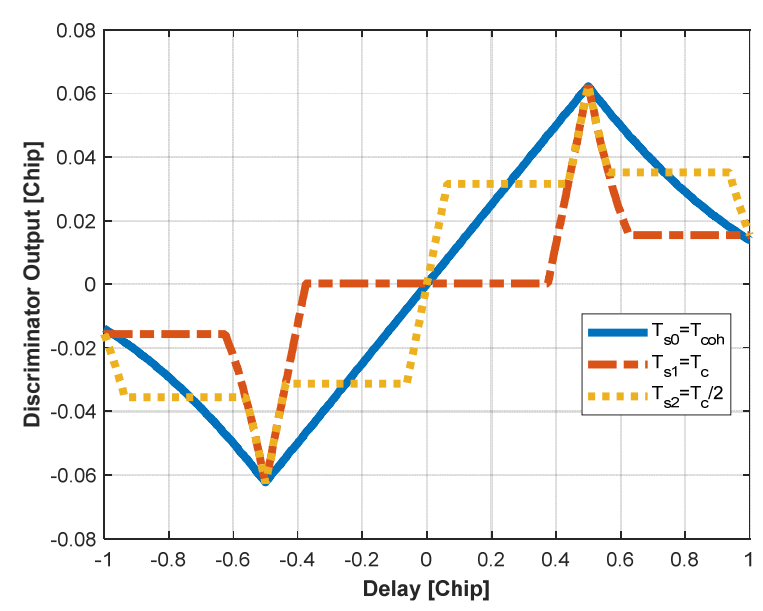

Fig. 10. S-curve of the code tracking loop when $d=1, d_{\mathrm{f}}=25 \%$, Phase II. 


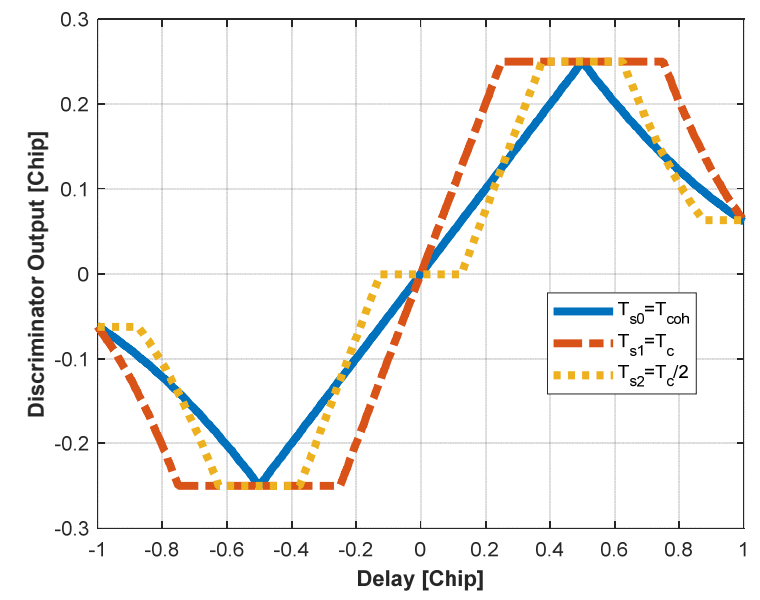

Fig. 11. S-curve of the code tracking loop when $d=1, d_{\mathrm{f}}=50 \%$, Phase I.

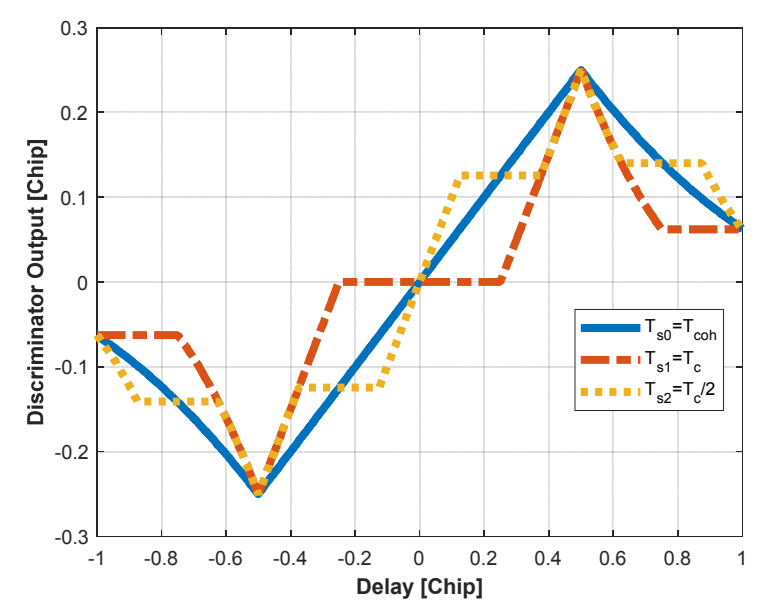

Fig. 12. S-curve of the code tracking loop when $d=1, d_{\mathrm{f}}=50 \%$, Phase II.

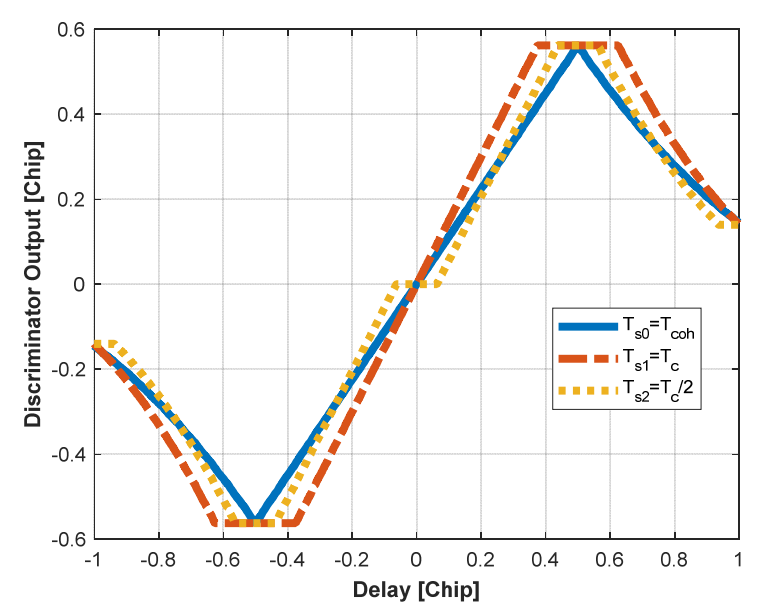

Fig. 13. S-curve of the code tracking loop when $d=1, d_{\mathrm{f}}=75 \%$, Phase I.

no deviation, which means the S-curve is crossing the zero point exactly of the coordinate, and when the pulse period is consistent with the code chip width and the pulse can keep the step part of the code wave, the slope of the Scurve is greater than that when the pulse period is consistent with the coherent integration time $T_{\text {coh }}$. In addition, the traction range of the discriminator is getting smaller when the pulse period is consistent with the code chip width.

When the correlator interval is $1 / 16$ chip, the pulse period is $T_{\mathrm{s} 0}=T_{\text {coh }}, T_{\mathrm{s} 1}=T_{\mathrm{c}}$ and $T_{\mathrm{s} 2}=T_{\mathrm{c}} / 2$, and the duty cycle is $25 \%, 50 \%$ and $75 \%$, respectively. The S-curves of two types of pulse phase are shown from Fig. 15 to Fig. 20.

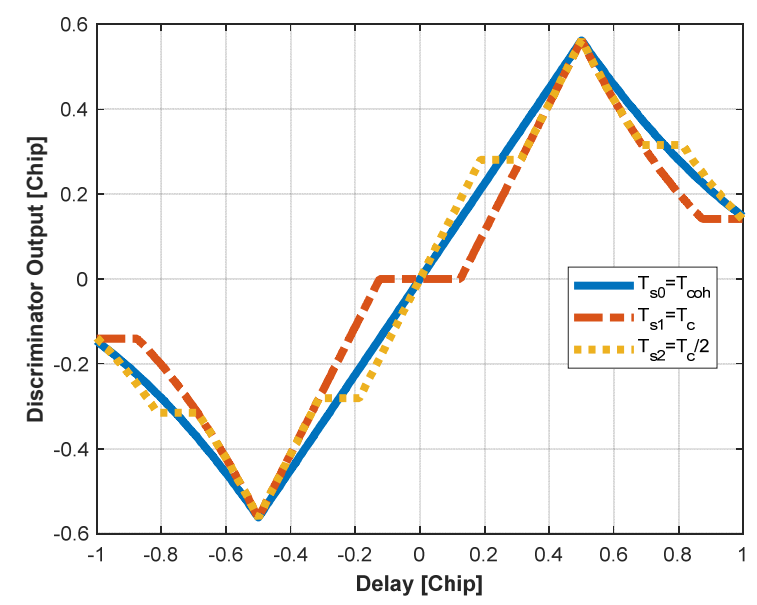

Fig. 14. S-curve of the code tracking loop when $d=1, d_{\mathrm{f}}=75 \%$, Phase II.

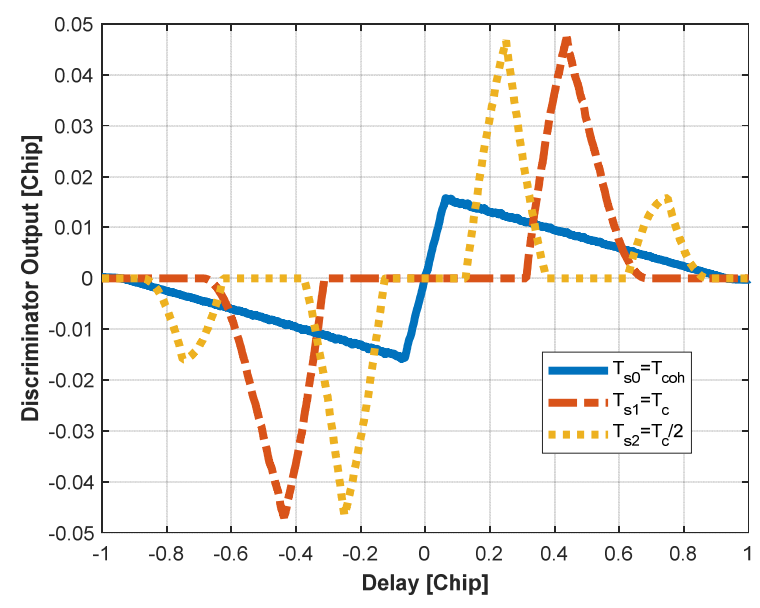

Fig. 15. S-curve of the code tracking loop when $d=1 / 8$, $d_{\mathrm{f}}=25 \%$, Phase I.

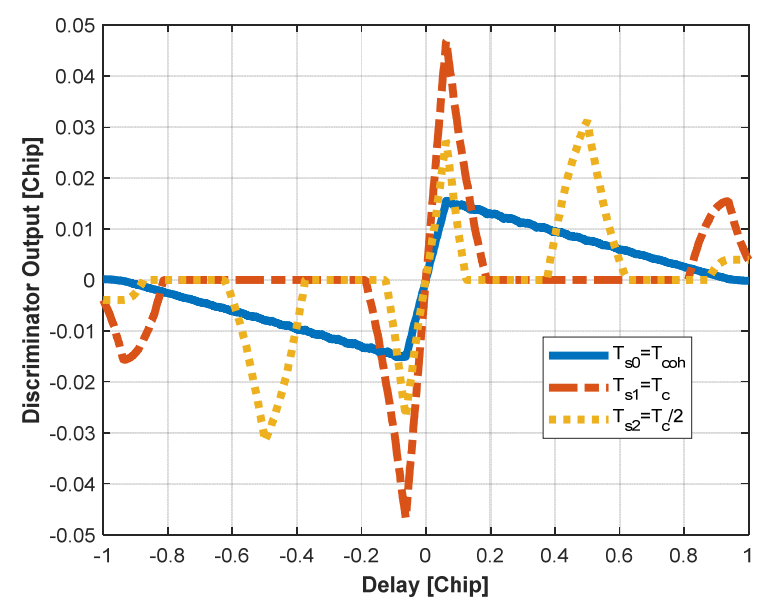

Fig. 16. S-curve of the code tracking loop when $d=1 / 8$, $d_{\mathrm{f}}=25 \%$, Phase II. 


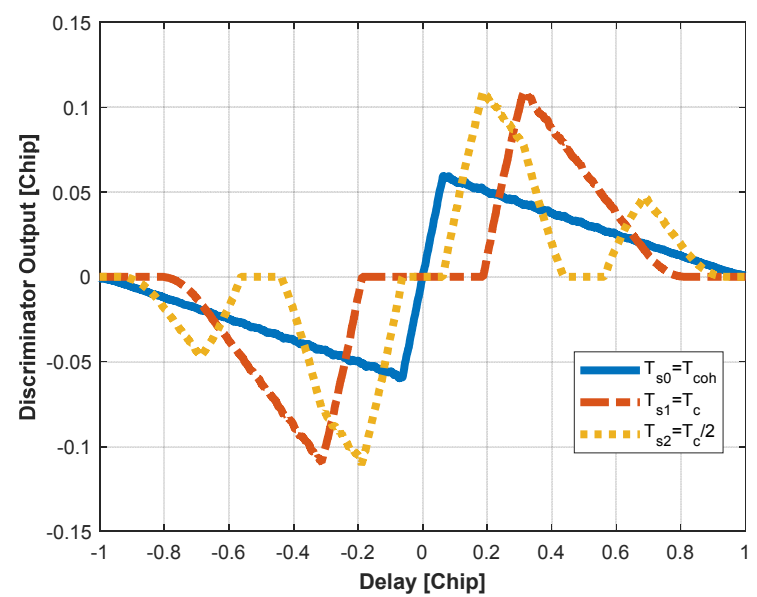

Fig. 17. S-curve of the code tracking loop when $d=1 / 8$, $d_{\mathrm{f}}=50 \%$, Phase I.

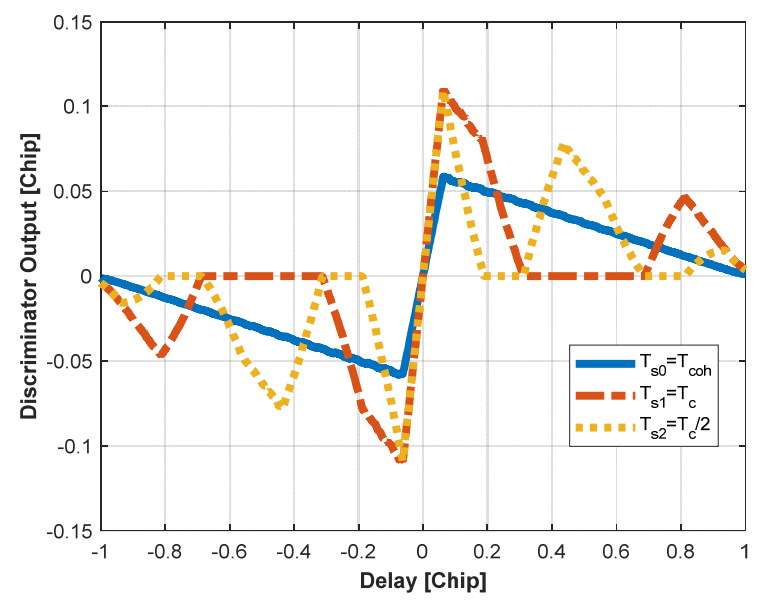

Fig. 18. S-curve of the code tracking loop when $d=1 / 8$, $d_{\mathrm{f}}=50 \%$, Phase II.

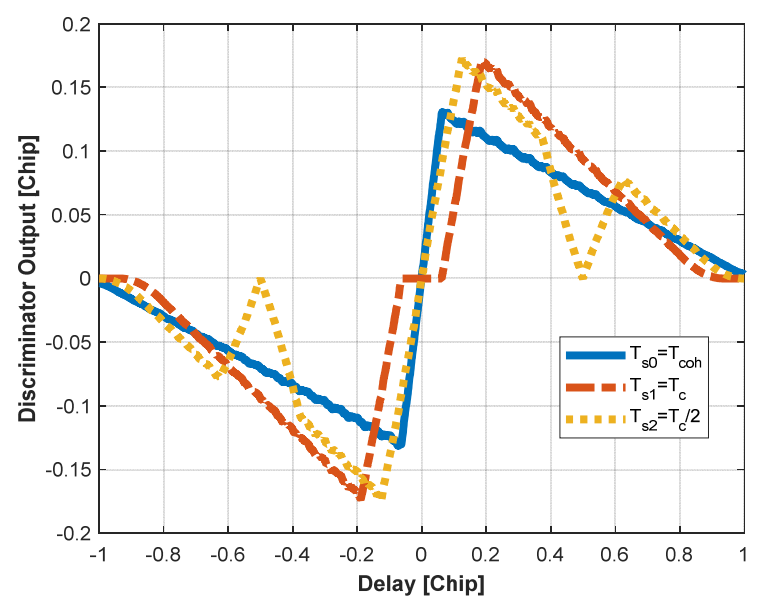

Fig. 19. S-curve of the code tracking loop when $d=1 / 8$, $d_{\mathrm{f}}=75 \%$, Phase I.

As can be seen from Fig. 15 to Fig. 20, although the slope increases and the narrow correlation can reduce the ranging deviation, more zero-crossing points will be introduced. If the tracking results converge to any zero-crossing point, there will be a larger ranging deviation.

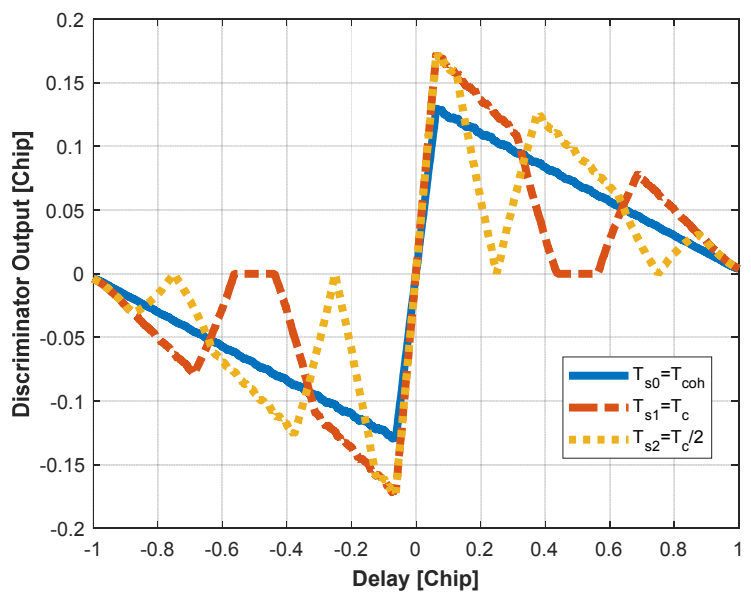

Fig. 20. S-curve of the code tracking loop when $d=1 / 8$, $d_{\mathrm{f}}=75 \%$, Phase II.

\subsection{Code Tracking Accuracy}

The simulation verification of code tracking accuracy is carried out in the form of non-coherent processing. The number of Monte Carlo simulations is $10^{6}$ and the time consumptions of each simulation are about 4.988 hours. The signal GPS L1CA is selected and the simulation settings are shown in Tab. 3 and the simulation flow chart is shown as Fig. 21.

When the correlator interval is $1 / 2$ chip, the simulation result of tracking accuracy by non-coherent processing is shown as Fig. 22. As can be seen from Fig. 22, when the pulse period $T_{\mathrm{s} 1}=T_{\text {coh }}$, the code tracking accuracy decreases as expected. However, when the pulsed period $T_{\mathrm{s} 2}=T_{\mathrm{c}}$ and the pulse phase is the first type (Phase I), the code tracking accuracy under blanking is even higher than that of the continuous wave, and the pulse blanking plays the role of narrow correlation.

When the correlator interval is $1 / 16$ chip, the simulation results of tracking accuracy by non-coherent processing are shown as Fig. 23. As can be seen from Fig. 23, when the pulsed period $T_{\mathrm{s} 1}=T_{\text {coh }}$, the code tracking accuracy decreases as expected, which is the same as the situation when the correlator interval is $1 / 2$ chip. However, when the pulse period $T_{\mathrm{s} 2}=T_{\mathrm{c}}$ and the pulse signal phase is the second type (Phase II), the code tracking accuracy under

\begin{tabular}{|c|c|c|}
\hline No. & Parameters & Values \\
\hline 1 & Sampling rate & $5.5 \mathrm{MHz}$ \\
\hline 2 & Integration time & $1 \mathrm{~ms}$ \\
\hline 3 & Signal length & $1 \mathrm{~s}$ \\
\hline 4 & Doppler & $100 \mathrm{~Hz}$ \\
\hline 5 & Correlator interval & $1 / 2,1 / 16$ \\
\hline 6 & C/N $\mathrm{N}_{0}$ & $40 \mathrm{dBHz}$ to $50 \mathrm{dBHz}$ \\
\hline 7 & Pulse signal period & $T_{\mathrm{s} 1}=T_{\text {coh, }} T_{\mathrm{s} 2}=T_{\mathrm{c}}$ \\
\hline 8 & Duty cycle & $50 \%$ \\
\hline 9 & Code loop order & 2 \\
\hline 10 & Code loop bandwidth & $1 \mathrm{~Hz}$ \\
\hline 11 & Carrier loop order & 3 \\
\hline 12 & Carrier loop bandwidth & $18 \mathrm{~Hz}$ \\
\hline
\end{tabular}

Tab. 3. Code tracking simulation parameters. 


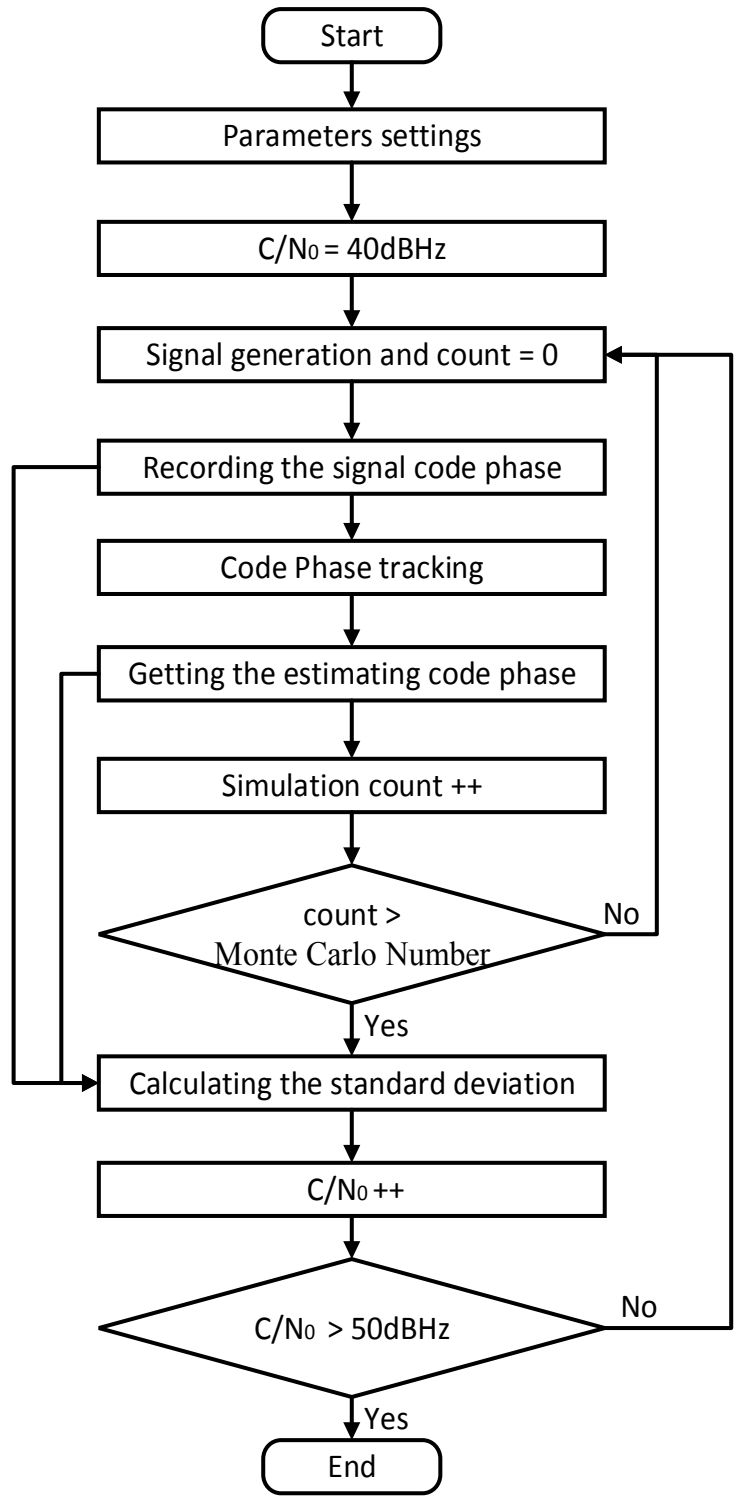

Fig. 21. The simulation flow chart of code tracking accuracy.

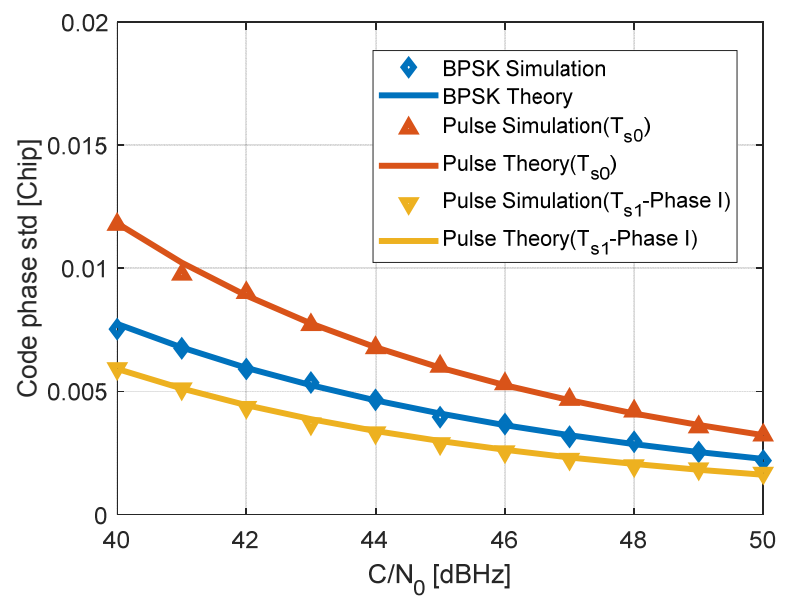

Fig. 22. Code tracking accuracy when $d=1$.

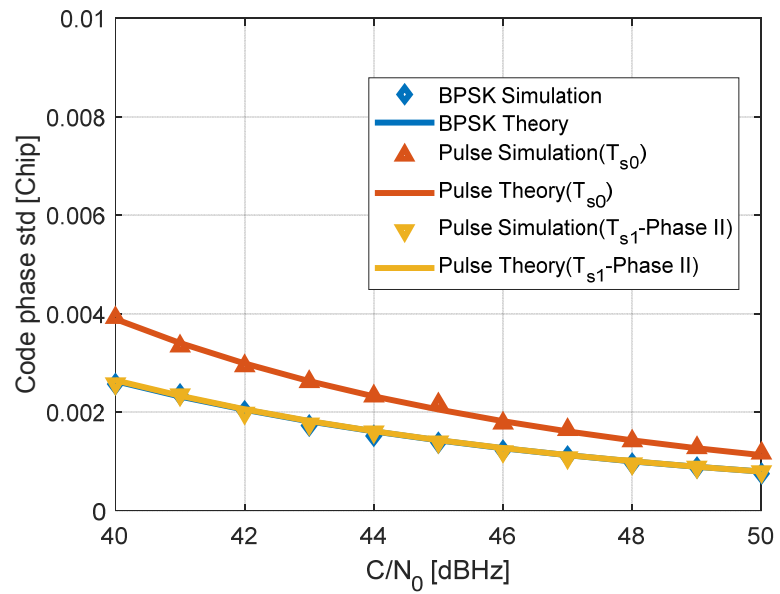

Fig. 23. Code tracking accuracy when $d=1 / 8$.

blanking is the same as that of the continuous wave. This is because the power of the noise component and the high frequency part of the signal are the same in the tracking loops under these two situations.

In the actual receiver processing, the time relationship between the initial phase of the pulse gate and the initial accumulation time of the integrator is random after the ideal pulse blanking. It is very likely that the tracking results in different channels are different. The tracking accuracy will not decrease significantly in some channels. However, the tracking accuracy will decrease or there will be a large deviation in the tracking process of other channels.

\section{Conclusion}

Pulse interference, as one of the most common nonstationary interference, has always been a major threat to the satellite navigation receiver. The blanking method can effectively mitigate the impact of pulse interference. This paper focuses on the effects of the blanking method on receiver performance of code tracking.

The work in this paper mainly makes up for the lack of performance evaluation of the GNSS receiver with blanking model under pulse interference with different duty cycle and different periods. The code tracking deviation accuracy is paid more attention in this paper, and the signal model established in this work and the evaluation results extend the current researches. It can be found through theoretical analysis and simulation verification that it is likely to cause a large tracking deviation except for causing a decline in tracking accuracy in the code tracking stage, which will seriously affect the tracking performance of the receiver. On the other hand, the code tracking variance under thermal noise may not be bigger obviously under the condition of specific relative phase and correlator interval when the blanking operation plays a role of narrow correlation. 
In order to avoid ranging performance degradation, this work can be used as a reference for designing better algorithms to suppress pulse interference. In particular, how to restrain the pseudo-range deviation caused by pulse interference blanking needs to be solved in the future work.

\section{References}

[1] LIU, Y., RAN, Y., KE, T., et al. Code tracking performance analysis of GNSS signal in the presence of CW interference. Signal Processing, 2011, vol. 91, no. 4, p. 970-987, DOI: 10.1016/j.sigpro.2010.09.022

[2] GAO, G. X. DME/TACAN interference and its mitigation in L5E5 bands. In Proceedings of the 20th International Technical Meeting of the Satellite Division of the Institute of Navigation. Fort Worth (TX, USA), September 2007, p. 1191-1200.

[3] BORIO, D., O'DRISCOLL, C., FORTUNY-GUASCH, J. Pulsed pseudolite signal effects on non-participating GNSS receivers. In Proceedings of International Conference on Indoor Positioning and Indoor Navigation. Guimaraes (Portugal), September 2011, p. 1-6. DOI: 10.1109/IPIN.2011.6071912

[4] PARKINSON, B. W., ENGE, P., AXELRAD, P., et al. Global Positioning System: Theory and Applications. Vol. I. Washington, DC (USA): AIAA, 1996. ISBN: 1-56347-106-X

[5] DOVIS, F. GNSS Interference Threats and Countermeasures. Norwood, (MA USA): Artech House, 2015. ISBN: 978-1-60807810-3

[6] KAPLAN, E. D., HEGARTY, C. Understanding GPS: Principles and Applications. $2^{\text {nd }}$ ed. Norwood, (MA, USA): Artech House, 2005. ISBN:978-7-121-04584-4

[7] FANG, W., WU, R. WANG, W., et al. DME pulse interference suppression based on NLS for GPS. In Proceedings of 2012 IEEE 11th International Conference on Signal Processing. Beijing (China), October 2012, p. 174-178. DOI: 10.1109/ICoSP.2012.6491629

[8] ANYAEGBU, E., BRODIN, G., COOPER, J., et al. An integrated pulsed interference mitigation for GNSS receivers. Journal of Navigation, 2008, vol. 61, no. 2, p. 239-255, DOI: $10.1017 / \mathrm{s} 0373463307004572$

[9] LI, L., WANG, W., LU, D., et al. Wavelet packet transformation based technique in mitigation DME pulsed interference for GNSS. In Proceedings of China Satellite Navigation Conference. Nanjing (China), May 2014, p. 715-724. DOI: 10.1007/978-3-642-547379_62

[10] GAO, G. X., SGAMMINI, M., LU, M., et al. Protecting GNSS receivers from jamming and interference. Proceedings of the IEEE, 2016, vol. 104, no. 6, p. 1327-1338, DOI: 10.1109/JPROC.2016.2525938

[11] HEGARTY, C., VAN DIERENDONCK, A. J., BOBYN, D., et al. Suppression of pulsed interference through blanking. In Proceedings of the IAIN World Congress and the 56th Annual Meeting of the Institute of Navigation. San Diego (CA, USA), June 2000, p. 399-408.

[12] GRABOWSKI, J., HEGARTY, C. Characterization of L5 receiver performance using digital pulse blanking. In Proceedings of the 15th International Technical Meeting of the Satellite Division of the Institute of Navigation. Portland (OR, USA), September 2002, p. $1630-1635$.

[13] BORIO, D., CANO, E. Optimal Global Navigation Satellite System pulse blanking in the presence of signal quantization. IET
Signal Processing, 2013, vol. 7, no. 5, p. 400-410. DOI: 10.1049/iet-spr.2012.0199

[14] GARCIA-PENA, A., JULIEN, O., GAKNE, P. V., et al. Efficient DME/TACAN blanking method for GNSS-based navigation in civil aviation. In Proceedings of the 32nd International Technical Meeting of the Satellite Division of the Institute of Navigation. Miami (FL, USA), September 2019, p. 1438-1452. DOI: $10.33012 / 2019.16993$

[15] HUO, S., NIE, J., WANG, F. Block-flow noise power estimation algorithm for pulsed interference detection of GNSS receivers. Electronics Letters, 2015, vol. 51, no. 19, p. 1522-1524. DOI: 10.1049/el.2015.1445

[16] MUSUMECI, L., SAMSON, J., DOVIS, F. Performance assessment of pulse blanking mitigation in presence of multiple Distance Measuring Equipment/Tactical Air Navigation interference on Global Navigation Satellite Systems signals. IET Radar, Sonar \& Navigation, 2014, vol. 8, no. 6, p. 647-657. DOI: 10.1049/iet-rsn.2013.0198

[17] GARCIA-PENA, A., MACABIAU, C., JULIEN, O., et al. Impact of DME/TACAN on GNSS L5/E5a receiver. In Proceedings of the 2020 ITM. San Diego (CA, USA), January 2020, p. 207-211. DOI: $10.33012 / 2020.17207$

[18] BEK, M. K., SHAHEEN, E. M., ELGAMEL, S. A. Mathematical analyses of pulse interference signal on post-correlation carrier-tonoise ratio for the global positioning system receivers. IET Radar, Sonar \& Navigation, 2015, vol. 9, no. 3, p. 266-275. DOI: 10.1049/iet-rsn.2014.0155

[19] BASTIDE, F., CHATRE, E., MACABIAU, C., et al. GPS L5 and GALILEO E5a/E5b signal-to-noise density ratio degradation due to DME/TACAN signals simulations and theoretical derivation. In Proceedings of the 2004 National Technical Meeting of the Institute of Navigation. San Diego (CA, USA), January 2004, p. 1049-1062.

[20] MUSUMECI, L., DOVIS, F. Effect of pulse blanking on navigation data demodulation performance in GNSS system. In Proceedings of 2014 IEEE/ION Position, Location and Navigation Symposium - PLANS 2014. Monterey (CA, USA), May 2014, p. 1248-1257. DOI: 10.1109/PLANS.2014.6851500

[21] YESTE OJEDA, O. A., GRAJAL, J., LOPEZ-RISUENO, G. Analytical performance of GNSS receivers using interference mitigation techniques. IEEE Transactions on Aerospace and Electronic Systems, 2013, vol. 49, no. 2, p. 885-906. DOI: $10.1109 /$ taes.2013.6494387

[22] BETZ, J. W., KOLODZIEJSKI, K. R. Generalized theory of code tracking with an early-late discriminator. Part I: Lower bound and coherent processing. IEEE Transactions on Aerospace and Electronic Systems, 2009, vol. 45, no. 4, p. 1538-1556. DOI: $10.1109 /$ taes.2009.5310316

[23] BETZ, J. W., KOLODZIEJSKI, K. R. Generalized theory of code tracking with an early-late discriminator. Part II: Noncoherent processing and numerical results. IEEE Transactions on Aerospace and Electronic Systems, 2009, vol. 45, no. 4, p. 1557-1564. DOI: $10.1109 /$ taes.2009.5310317

[24] HOLMES, J. K. Spread Spectrum Systems for GNSS and Wireless Communications. Norwood (MA, USA), 2007. ISBN: 978-7-12118082-8

[25] STANSELL, T. A. RTCM SC-104 Recommended pseudolite signal specification. Navigation, 1986, vol. 33, no. 1, p. 42-59. DOI: $10.1002 / j .2161-4296.1986 . t b 00923 . x$

[26] BORIO, D., O'DRISCOLL, C. Design of a general pseudolite pulsing scheme. IEEE Transactions on Aerospace and Electronic Systems, 2014, vol. 50, no. 1, p. 2-16. DOI: $10.1109 /$ taes.2013.110277 


\section{About the Authors ...}

Pengcheng MA was born in 1992. He is a Ph.D. candidate in the College of Electronic Science and Technology at the National University of Defense Technology (NUDT) in China. He received his M.Sc. from the NUDT in 2016. His research interests include GNSS signal processing and antijamming techniques.

Xiaomei TANG was born in 1982. She received her M.S. and Ph.D. from the College of Electronic Science and Technology, National University of Defense Technology (NUDT) in China. She majors in GNSS signal acquisition and tracking.

Shengqiang LOU (corresponding author) was born in
1969. He is an Associate Professor at the National University of Defense Technology, where he received his Ph.D. degree from the College of Electronic Science and Technology. He interests in GNSS signal processing.

Kuixing LIU was born in 1983. He is a senior engineer in Beijing Satellite Navigation Center. His research interests include satellite navigation engineering.

Gang OU was born in 1969 and received the M.S. and Ph.D. degrees from the College of Electronic Science and Technology, National University of Defense Technology. $\mathrm{He}$ is a Professor, majoring in Global Navigation Satellite System signal processing, data processing, and antijamming methods for GNSS. 